\title{
Antimony resistance in Leishmania (Viannia) braziliensis clinical isolates from atypical lesions associates with increased ARM56/ARM58 transcripts and reduced drug uptake
}

\author{
Jeronimo Nunes Rugani', Célia Maria Ferreira Gontijo', Frédéric Frézard², \\ Rodrigo Pedro Soares ${ }^{1}$, Rubens Lima do Monte-Neto ${ }^{1 /+}$ \\ ${ }^{1}$ Fundação Oswaldo Cruz-Fiocruz, Instituto René Rachou, Belo Horizonte, MG, Brasil \\ ${ }^{2}$ Universidade Federal de Minas Gerais, Instituto de Ciências Biológicas, Departamento de Fisiologia e Biofísica, Belo Horizonte, MG, Brasil
}

BACKGROUND In addition to the limited therapeutic arsenal and the side effects of antileishmanial agents, drug resistance hinders disease control. In Brazil, Leishmania braziliensis causes atypical (AT) tegumentary leishmaniasis lesions, frequently refractory to treatment.

OBJECTIVES The main goal of this study was to characterise antimony (Sb)-resistant (SbR) L. braziliensis strains obtained from patients living in Xakriabá indigenous community, Minas Gerais, Brazil.

METHODS The aquaglyceroporin 1-encoding gene (AQP1) from L. braziliensis clinical isolates was sequenced, and its function was evaluated by hypo-osmotic shock. mRNA levels of genes associated with Sb resistance were measured by quantitative reverse transcription polymerase chain reaction (qRT-PCR). Atomic absorption was used to measure Sb uptake.

FINDINGS Although clinical isolates presented delayed recovery time in hypo-osmotic shock, AQP1 function was maintained. Isolate 340 accumulated less $\mathrm{Sb}$ than all other isolates, supporting the 65 -fold downregulation of AQP1 mRNA levels. Both 330 and 340 isolates upregulated antimony resistance marker (ARM) 56/ARM58 and multidrug resistant protein A (MRPA); however, only ARM58 upregulation was an exclusive feature of SbR field isolates. CA7AE seemed to increase drug uptake in $L$. braziliensis and represented a tool to study the role of glycoconjugates in Sb transport.

MAIN CONCLUSIONS There is a clear correlation between ARM56/58 upregulation and Sb resistance in AT-harbouring patients, suggesting the use of these markers as potential indicators to help the treatment choice and outcome, preventing therapeutic failure.

Key words: antimony resistance - Leishmania braziliensis - field isolates - antimony resistance marker - ARM56 - ARM58 - atypical lesions

Leishmania (Viannia) braziliensis causes cutaneous leishmaniasis (CL), severe mucocutaneous leishmaniasis (MCL), and unusual cutaneous manifestations. Unlike typical CL lesions - commonly classified by a well-defined and limited oval ulcer, atypical (AT) tegumentary leishmaniasis, is recognised by dry, verrucous, and sporotrichoid lesions, which leads to misdiagnosis due to similarities with other dermatological pathologies. ${ }^{(1,2,3)}$ In Brazil, AT lesions were reported in Minas Gerais and Bahia states..$^{(1,3,4)}$ The histopathology of AT lesions caused by L. braziliensis, similar to classical CL lesions, shows infiltrated plasma cells and lymphocytes with few or none parasites. ${ }^{(1)}$ AT and CL lesions present similar inflammatory cytokine levels, differing only if the lesion has been formed more recently or later. In more recent lesions, there is high expression of a panel

doi: 10.1590/0074-02760190111

Financial support: CNPq (grant numbers: 310640/2017-2, 301597/2013-8 and 305430/2017-3), CAPES (001).

RPS, RLMN, CMG and FF are research fellows from CNPq. RLMN is a

grantee of Instituto Serrapilheira (grant number \#3660).

+ Corresponding author: rubens.monte@fiocruz.br

(D) https://orcid.org/0000-0002-4688-2462

Received 29 March 2019

Accepted 12 July 2019 of pro-inflammatory cytokines, while modulatory cytokines are characteristic of later lesions. ${ }^{(2,5)}$ In addition, $L$. braziliensis AT-derived clinical isolates are associated with a variant genetic profile, showing a clustered distribution in population structure analysis. ${ }^{(4)}$

We have recently observed that AT lesion-derived $L$. braziliensis strains are highly resistant to antimony (Sb), supporting the treatment failure observed in L. braziliensis $\mathrm{Sb}$-resistant (SbR) infected patients who received glucantime as chemotherapy. ${ }^{(3)} \mathrm{Sb}$ resistance was first described in India for Leishmania donovani-causing visceral leishmaniasis (VL), ${ }^{(6)}$ and treatment refractory CL due to species from the $L$. (Viannia) subgenus, including $L$. braziliensis, were reported in South America. ${ }^{(2,7)}$ L. braziliensis is commonly more sensitive to $\mathrm{Sb}$ than other species. However, L. braziliensis isolated from patients with treatment failure history derived from both CL typical or AT lesions were found to be less sensitive to treatment compared to isolates from patients with clinical cure. ${ }^{(3)}$

To avoid the spread of drug resistance, $\mathrm{Sb}$ resistant strains should be identified before treatment is chosen and administered. Therefore, the molecular mechanisms associated with the resistance phenotype in field isolates should be characterised so that select key markers can be screened for drug resistance in the field. In Leishmania parasites, $\mathrm{Sb}$ resistance is due to decreased drug uptake, increased drug efflux, or drug neutralisation strategies that pump active drug in intracellular vesicles 
or inactivate the drug through thiol binding followed by $\mathrm{Sb}$ extrusion. ${ }^{(8)}$ Leishmania parasites can become resistant to $\mathrm{Sb}$ by reducing expression or mutating the aquaglyceroporin 1-encoding gene (AQP1). This porin is the main entry route of $\mathrm{Sb}$ into the cell, and its disruption - achieved by gene deletion or point mutation - culminates with non-functional $\mathrm{AQP} 1$ unable to mediate $\mathrm{Sb}$ uptake and intracellular $\mathrm{Sb}$ accumulation. A similar effect can also be observed upon downregulation of AQP1 leading to diminished $\mathrm{Sb}$ levels within the parasite..$^{(9,10,11)}$ The same phenotype is reached when $\mathrm{Sb}$ is a substrate of the $\mathrm{ABC}$ transporters and can be pumped out or transported into intracellular vesicles, followed by extrusion. Several transporter proteins were associated with $\mathrm{Sb}$ resistance in different Leishmania species, from both field isolates and laboratory-selected resistant mutants such as, multidrug resistant protein A (MRPA)/ABCC3 (also known as PGPA), ${ }^{(12,13)} \mathrm{ABCG} 2{ }^{(14)} \mathrm{ABCI} 4{ }^{(15)}$ and antimony resistance marker (ARM)56/ARM58. ${ }^{(16)}$ However, the roles of such resistance genes are still unknown for L. braziliensis isolates derived from AT lesions.

Here, we explored molecular markers associated with $\mathrm{Sb}$ resistance in L. braziliensis clinical isolates from an Indigenous community in the north of Minas Gerais state, Brazil. Sb-resistant strains were obtained from AT-harbouring patients refractory to glucantime treatment. Functional analysis, involving drug transport and osmoregulation, was performed in order to validate and characterise previously identified $\mathrm{Sb}$ resistance.

\section{MATERIALS AND METHODS}

Parasites - Promastigote forms of L. (Viannia) braziliensis isolated from classical CL lesion (strains MHOM/BR/2008/426 and MHOM/BR/2009/346), derived from AT lesions (strains MHOM/BR/2008/330 and $\mathrm{MHOM} / \mathrm{BR} / 2008 / \mathbf{3 4 0}$ ), reference strain $\mathrm{MHOM} /$ $\mathrm{BR} / 1975 / \mathbf{M 2 9 0 3}$, and L. (Leishmania) infantum $\Delta l p g$ (strain MCAN/BR/1989/BA262) $^{(17)}$ were maintained in a-MEM medium (Gibco/Thermo Fisher) supplemented with $10 \%$ foetal bovine serum, $5 \mu \mathrm{g} / \mathrm{mL}$ of hemin, and $5 \mu \mathrm{M}$ of biopterin in $\mathrm{pH} 7$ at $26^{\circ} \mathrm{C}$. Field isolates were previously typed as $L$. braziliensis. ${ }^{(3)} \mathrm{All}$ clinical isolates were obtained prior to patient treatment, meaning that isolates from AT lesions were intrinsically resistant to $\mathrm{Sb}^{(4)}$ The resistance was not acquired during Sb exposition in these patients. Patients were submitted to one round of treatment with $20 \mathrm{mg} \mathrm{Sb}^{\mathrm{V}}$ (glucantime as source of $\left.\mathrm{Sb}^{\mathrm{v}}\right) / \mathrm{kg}$ /day for 20 days. The patients who presented AT lesions were refractory and submitted to a second round of treatment to achieve cure. One of the patients - from whom the strain 340 was isolated - dropped out the therapeutic scheme. ${ }^{(3)}$ The resistance index of AT lesion-derived L. braziliensis was > 12-fold when compared to reference strain M2903. ${ }^{(3)}$

Hypo-osmotic shock assay - Hypo-osmotic stress was applied as a way to assess cell volume measurements and infer about osmoregulation, as previously described. ${ }^{(18)}$ Briefly, mid-log phase L. braziliensis promastigotes were washed twice in HEPES/NaCl/Glucose buffer (20 mM HEPES, $0.15 \mathrm{M} \mathrm{NaCl}, 10 \mathrm{mM}$ glucose, $\mathrm{pH}$ 7.2), adjusted to a final volume of $100 \mathrm{~mL}$ containing $1 \times 10^{8}$ parasites $/ \mathrm{mL}$, and incubated in a 96-well flat bottom plate. Hypo-osmotic shock was induced by adding equal volume of Milli-Q water to the isotonic parasite culture, followed by absorbance measurement at $550 \mathrm{~nm}$ during 5 min with $9 \mathrm{~s}$ interval between reads in a microplate reader (Spectramax M5, Molecular Devices). The culture dilution leads to changes in osmolarity (cellular hypo-osmotic stress) leading the cellular volume increase that corresponds to a reduction in absorbance. Isosmotic control was established by adding equal volume of saline buffer to the cultures. Absorbance measures were plotted using GraphPad Prism version 6.0 as normalised values by subtracting first read value from all time point reads. The experiments were performed three times in triplicate.

AQP1 sequencing - Genomic DNA of mid-log growth phase $L$. braziliensis promastigotes was extracted using Gentra Puregene Cell and Tissue Kit (QIAGEN \#158667) according to manufacturer's instructions. Genomic DNA was used to amplify AQP1 (LbrM.31.0020) by PCR using the following forward and reverse primers: 5'-ATGGCGATTGAAAACCACATGG-3' and 5'-CTACGCACCGCTCGGTATTATA-3', resulting in an amplicon that comprises the whole ORF of $858 \mathrm{bp}$. The resulted amplicon was purified using QIAquick polymerase chain reaction (PCR) purification kit (QIAGEN \#28104), cloned into pGEM-T easy vector (Promega, Madison, WI, USA), and Sanger sequenced in a capillary sequencer ABI3730xl DNA Analyzer (ThermoFischer) using SP6 (ATTTAGGTGACACTATAG) and T7 (TAATACGACTCACTATAGGG) primers. All sequences were analysed using FinchTV software (Geospiza, INC.), and amplicon sequences were aligned with sequences obtained from GenBank using the BLAST tool. ${ }^{(8)}$ The consensus sequences were aligned with L. braziliensis M2904 AQP1 gene (LbrM.31.0020) using MEGA X software to analyse the presence of single nucleotide polymorphisms (SNPs) and possible amino acid substitutions.

Intracellular antimony measurement - The Sb uptake assay was performed as previously reported. ${ }^{(19)}$ Mid-log growth phase Leishmania promastigotes were washed with HEPES/NaCl/Glucose buffer, and adjusted to a population of $1 \times 10^{8}$ cells $/ \mathrm{mL}$. Parasites $\left(1 \times 10^{8}\right)$ were incubated with $500 \mu \mathrm{M}$ of $\mathrm{Sb}^{\mathrm{III}}$ (potassium antimonyl tartrate, Sigma Aldrich \#244791) for $1 \mathrm{~h}$ at $26^{\circ} \mathrm{C}$ in a 24 -well plate. The cultures were transferred to microtubes, placed in ice for $\mathrm{Sb}$ transport blocking, and washed four times with 1 $\mathrm{mL}$ of HEPES/ $\mathrm{NaCl} / \mathrm{Glucose}$ ice-cold buffer at $1816 \mathrm{~g}$ for $5 \mathrm{~min}$ at $4^{\circ} \mathrm{C}$. The resulting pellet was digested overnight with $100 \mu \mathrm{L}$ of $65 \% \mathrm{HNO}_{3}$ (Merck Millipore \#100456). Intracellular $\mathrm{Sb}$ trace content was quantified by graphite furnace electrothermal atomic absorption spectrometry using an AAnalyst 600/800 spectrometer (Perkin Elmer, Waltham, MA, USA). Blank matrix was established as promastigotes without drug exposition, while the adsorption control was exposed to $\mathrm{Sb}$ at $4^{\circ} \mathrm{C}$ and washed immediately after drug exposure. Blank measure values were subtracted as background and intracellular Sb content was normalised by the number of cells. To perform the uptake kinetics, we incubated the parasites with the CA7AE antibody $(1: 400)$, which recognises the $\operatorname{Gal}(\beta 1,4)$ 
$\operatorname{Man}(\alpha 1)-\mathrm{PO}_{4}$ repeat units present in units present in lipophosphoglycan (LPG) surface molecule. All assays were performed three times as independent experiments with four replicates each.

Real-time reverse transcription PCR (RT-PCR) - Total RNA was extracted from mid-log phase promastigotes, using the RNeasy Plus Mini Kit (Qiagen \#74134), where $5 \mu \mathrm{g}$ was used to synthesise cDNA following manufacturer's instructions of Super-Script First Strand Synthesis System for RT-PCR (ThermoFischer \#11904018). This cDNA was used as a template in reactions containing $1 \times$ iTaq Universal SYBR Green Supermix (Bio-Rad \#10000068167), $100 \mathrm{nM}$ forward and reverse primers, and $100 \mathrm{ng}$ of cDNA target. The reaction was carried out in a program of $95^{\circ} \mathrm{C}$ for $5 \mathrm{~min}$, and then cycled 40 times at $95^{\circ} \mathrm{C}$ for $15 \mathrm{~s}$ and $60^{\circ} \mathrm{C}$ for $30 \mathrm{~s}$. The assays were performed in three biological replicates. Relative amounts of PCR products generated from each primer set were determined based on the cycle threshold $(\mathrm{Ct})$ value and amplification efficiencies. Data were analysed using the comparative $2^{-\Delta \Delta C t}$ method, where GAPDH (glyceraldehyde-3-phosphate dehydrogenase) coding gene (LbrM.30.2950) was used as a housekeeping gene. All primers for targeted genes and their sequences are listed in Table I.
Ethics - The L. braziliensis clinical isolates were obtained from patients from an indigenous reserve as previously published. ${ }^{(4)}$ This main project was approved by CONEP ${ }^{\circ} 355 / 2008$ and FUNAI authorisation $n^{\circ} 149 /$ CGEP/08 to access indigenous territory.

\section{RESULTS}

L. braziliensis SbR field isolates are able to recover cellular volume upon hypo-osmotic stress but slower than reference strain - The hypo-osmotic shock assay is a suitable method to evaluate the integrity of aquaglyceroporin function on osmotic control; therefore, we applied this strategy to associate AQP1 defects with the $\mathrm{Sb}$ resistance mechanism in L. braziliensis field isolates. All strains were able to recover cellular volume upon hypo-osmotic stress (Fig. 1A-E). However, field isolates 346, 330, and 340 presented a delayed recovery time when compared with the M2903 reference strain (Fig. 1F). Based on hypo-osmotic shock assay, we were not able to differentiate among typical or AT lesion-derived strains. However, we clearly see at least a 3 -fold reduction in the area under curve from field isolates when compared with M2903 (Fig. 1F), which is directly proportional to the slope and explains the osmoregulation delay.

TABLE I

Primers used in quantitative real-time polymerase chain reaction (PCR)

\begin{tabular}{|c|c|c|c|c|}
\hline Target gene (ID) & Sequence 5'- 3' (Forward and reverse) & $\operatorname{Tm}$ & $\mathrm{GC} \%$ & Amplicon length (bp) \\
\hline \multirow{2}{*}{$\begin{array}{l}\text { GAPDH } \\
(\text { LbrM.30.2950) }\end{array}$} & CGACCAGGACCTTATTGGTAAA & 63.7 & 45.5 & \multirow{2}{*}{100} \\
\hline & CACCGTATCGTGCTTCATCT & 62.7 & 50 & \\
\hline \multirow{2}{*}{$\begin{array}{l}\text { MRPA } \\
(\text { LbrM.23.0280) }\end{array}$} & CTTCTCCATGTGCTCCACTATC & 62.6 & 50 & \multirow{2}{*}{103} \\
\hline & CACCTGCATCAGCTTGTAGTA & 60.5 & 47.6 & \\
\hline \multirow{2}{*}{$\begin{array}{l}\text { AQP1 } \\
(\text { LbrM.31.0020) }\end{array}$} & GGTATCACGACAGGTATCAACTC & 61.3 & 47.8 & \multirow{2}{*}{84} \\
\hline & CCAGAGCATGGCTGTGAATA & 63.8 & 50 & \\
\hline \multirow{2}{*}{$\begin{array}{l}\text { GSH1 } \\
(\text { LbrM.18.1700) }\end{array}$} & GGGTGGCTTCTATGGACTTATAC & 61.5 & 47.8 & \multirow{2}{*}{100} \\
\hline & CATGGACAGGAACCTCAAGTAG & 62.5 & 50 & \\
\hline \multirow{2}{*}{$\begin{array}{l}\text { ABCG2 } \\
(\text { LbrM.06.0080) }\end{array}$} & CTGAGTTTCCCGTGCAGATT & 64.2 & 50 & \multirow{2}{*}{107} \\
\hline & CACACCGCAGTAGTAGAAGAAC & 60.4 & 50 & \\
\hline \multirow{2}{*}{$\begin{array}{l}\text { ABCI4 } \\
(\text { LbrM.33.3540) }\end{array}$} & CTGTAGACGAAGCGGGTATTT & 62 & 47.6 & \multirow{2}{*}{135} \\
\hline & CTAGGCGATGAGACACCATAAC & 62.4 & 50 & \\
\hline \multirow{2}{*}{$\begin{array}{l}\text { AMR56 } \\
(\text { LbrM.20.0200) }\end{array}$} & GAGAACGGACAACCCAGTTT & 62.9 & 50 & \multirow{2}{*}{105} \\
\hline & CCACTTCTTCTGCTGTGAGTT & 61.1 & 47.6 & \\
\hline \multirow{2}{*}{$\begin{array}{l}\text { ARM58 } \\
(\text { LbrM.20.0210) }\end{array}$} & CCCAAGGGCTTTCACCTAAA & 64.9 & 50 & \multirow{2}{*}{103} \\
\hline & AGCGGTAGATCTTGTCGTATTG & 62 & 45.5 & \\
\hline
\end{tabular}

GAPDH: glycosomal glyceraldehyde 3-phosphate dehydrogenase; MRPA: multidrug resistant protein A (ABC transporter ABCC3, also known as PGPA); AQP1: aquaglyceroporin 1; GSH1: gamma-glutamylcysteine synthetase; ABCG2: ATP-binding cassette protein subfamily G, member 2; ABCI4: ATP-binding cassette protein subfamily I member 4; ARM56 and ARM58: antimony resistance marker of 56 and $58 \mathrm{kDa}$. bp: base pair. Tm (melting temperature) was calculated at default settings of $0.25 \mathrm{mM}$ oligo concentration and $50 \mathrm{mM} \mathrm{Na}$. Primers were designed using PrimerQuest (www.idtdna.com/site/account/login?returnurl=\%2FPrimerq uest $\% 2$ FHome $\% 2$ FIndex), rechecked with OligoAnalyzer (https://www.idtdna.com/calc/analyzer) and OligoEvaluator (Guimarães et al., ${ }^{(1,2)}$ Rugani et al. $\left.{ }^{(3)}\right)$. 
TABLE II

Single nucleotide polymorphisms (SNPs) of the AQP1 gene of Leishmania braziliensis field isolates and M2903 reference strain

\begin{tabular}{|c|c|c|c|}
\hline Strain & Lesion & DNA & Aminoacid \\
\hline \multirow{4}{*}{ M2903 } & \multirow{4}{*}{ Typical } & A $372 G$ & S \\
\hline & & T394C & S \\
\hline & & G447A & S \\
\hline & & $\mathrm{T} 449 \mathrm{C}$ & V150A \\
\hline \multirow{4}{*}{346} & \multirow{4}{*}{ Typical } & A372G & S \\
\hline & & T394C & S \\
\hline & & G447A & S \\
\hline & & T449C & V150A \\
\hline \multirow{4}{*}{426} & \multirow{4}{*}{ Typical } & A372G & $\mathrm{S}$ \\
\hline & & T394C & S \\
\hline & & G447A & S \\
\hline & & T449C & V150A \\
\hline \multirow{4}{*}{330} & \multirow{4}{*}{ Atypical } & A372G & S \\
\hline & & T394C & S \\
\hline & & T449C & $\mathrm{S}$ \\
\hline & & A516C & $\mathrm{S}$ \\
\hline \multirow{4}{*}{340} & \multirow{4}{*}{ Atypical } & A372G & S \\
\hline & & T394C & S \\
\hline & & T449C & S \\
\hline & & A516C & $\mathrm{S}$ \\
\hline
\end{tabular}

Red: SNP exclusive from AT lesion-derived L. braziliensis; blue: SNP present only in typical lesion-derived parasites and shared with M2903, but differs from reference genome of M2904; green: SNPs present in AQP1 from field isolates, shared with M2903, but absent in M2904. S: silent mutation. Primers AQP1F and AQP1R were used for amplification of fragment, and SP6 and T7 for sequencing.

SNPs in AQP1 gene coding sequence led to silent mutations and did not associate with SbR in L. braziliensis field isolates - Once we determined that the AQP1 main function was not disrupted, we decided to investigate its gene coding sequencing integrity, mRNA, and $\mathrm{Sb}$ uptake levels as an attempt to implicate or exclude this marker in SbR L. braziliensis field isolates. When comparing the AQP1 gene sequence of L. braziliensis field isolates with the M2903 and M2904 reference strains, we observed that AT lesion-derived SbR strains, 330 and 340, presented the SNP, A516C; however, this SNP did not alter the protein sequence and was considered a silent mutation [Table II, Supplementary data (Figure)]. The Guanine to Adenine change in the AQP1 gene at position 447 led to a V150A point mutation in the amino acid sequence from typical lesion-derived parasites (Table II). This feature was also shared with M2903, but differed in the M2904 reference strain. Three other SNPs, A372G, T394C, and T449C, were observed in M2903 and all field isolated strains [Table II, Supplementary data (Figure)].
SbR L. braziliensis field isolates upregulated ARM56/ARM58 while downregulating AQP1 - Several molecular markers have been associated with Sb resistance in Leishmania parasites. Because drug resistance is a multifactorial phenomenon, we decided to investigate the transcript level of not only AQP1, but also $\mathrm{Sb}$ resistance-associated targets, such as ARM56 and 58, MRPA, ABCI4, ABCG2, and GSH1. Downregulation in AQP1 was observed in SbR L. braziliensis isolates 330 and 340 (Fig. 2A), revealing that reduced Sb uptake could be associated with resistance mechanisms, even if its sequence is preserved. AQP1 was highly downregulated in isolate 340, reaching 65-fold when compared with M2903, while AQP1 mRNA levels of 330 were reduced 3-fold (Fig. 2A). Another clear difference in $\mathrm{Sb}$ resistance-associated genes is the upregulation of $\mathrm{Sb}$ resistance markers, ARM56/ARM58, respectively, in SbR AT lesion-derived L. braziliensis (Fig. 2B-C). ARM58 was exclusively upregulated in SbR parasites, reinforcing its chance of being directly involved in $\mathrm{Sb}$ resistance in these isolates (Fig. 2C). Upregulation of drug efflux pump, like ABCC3 (MRPA), was also observed in SbR parasites; however, it was not an exclusive feature of them, discouraging its association with resistance mechanism, because Sb-sensitive 346 upregulated this marker as well (Fig. 2D). Curiously, GSH1 encoding gene mRNA was downregulated in isolate 346 (Fig. 2E). This downregulation could explain Sb sensitivity, because thiol-mediated drug efflux or neutralisation was potentially incipient or absent due to reduced glutathione levels. Downregulation of ABCI4 would also support Sb susceptibility in L. braziliensis isolate 426 (Fig. 2F). Among the tested SbR markers, ABCG2 was the only one to remain non-modulated and non-associated with either the sensitivity or resistance mechanisms (Fig. 2G).

SbR L. braziliensis isolate 340 presented reduced Sb uptake - Because AQP1 mRNA was reduced in SbR AT lesion-derived L. braziliensis, we decided to measure and compare $\mathrm{Sb}$ uptake in all field isolates in order to functionally characterise Sb entry transport. Sb incorporation into L. braziliensis SbR isolate 340 was reduced when compared to $\mathrm{Sb}$-sensitive strains and even to its counterpart, isolate 330 (Fig. 3A), corroborating what was observed in mRNA expression results and functionally validating this mechanistical observation. Sb-sensitive isolate 346 presented a wide distribution in Sb uptake values among three independent experiments, reaching the highest intracellular Sb levels (Fig. 3A).

L. braziliensis surface glycoconjugates could interfere with Sb uptake - The heterogeneity and high levels of intracellular Sb content observed in Sb-sensitive $L$. braziliensis isolate 346 led us to further evaluate differential $\mathrm{Sb}$ accumulation for a better understanding of $\mathrm{Sb}$ uptake in Sb-sensitive isolates and potentially speculate about $\mathrm{Sb}$ transport-dependent drug resistance. For this analysis, we blocked the major Leishmania glycoconjugate LPG with CA7AE prior to Sb exposure. After $1 \mathrm{~h}$, we did not see any difference in Sb uptake (Fig. 3A-B), although adsorbed controls varied. Then, we decided to pursue an early time point kinetics (5-30 min). CA7AE- 

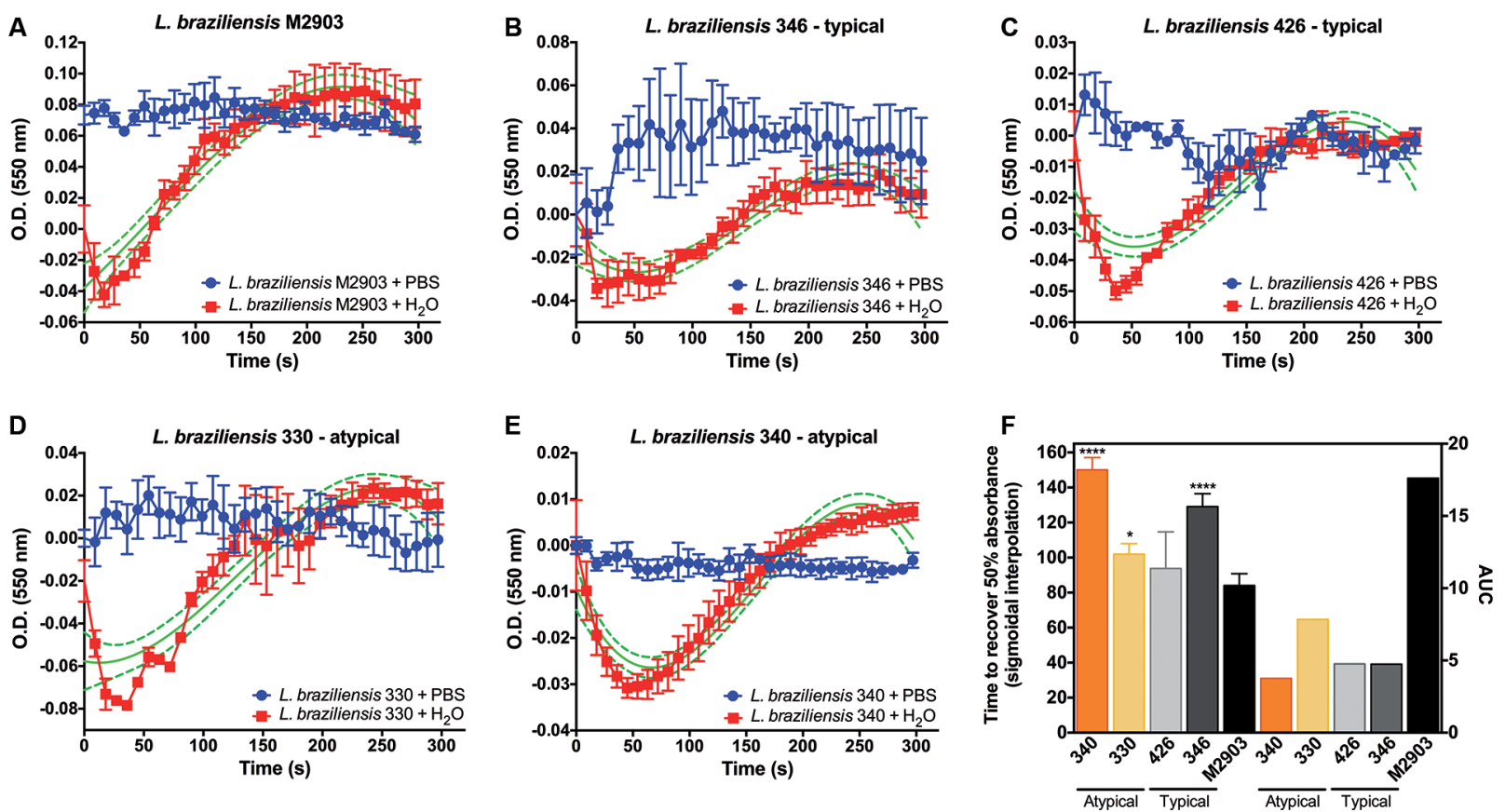

Fig. 1: volume regulation of Leishmania braziliensis field isolates. Hypo-osmotic shock was performed upon equal volume addition of Milli-Qwater to the cultures (red). Isotonic controls were established by adding isotonic saline buffer (blue). Absorbance was measured at $550 \mathrm{~nm}$ during $5 \mathrm{~min}$ after hypo-osmotic stress. Increases in cellular volume are represented by reduced absorbance, which is reverted by normal cellular osmoregulation. The recovery time was calculated using non-linear regression functions (sigmoidal, bell shaped, and third order polynomial). Sigmoidal interpolation is represented in green. The time to recover $50 \%$ in absorbance was compared using a one-way analysis of variance (ANOVA) followed by Bonferroni's multiple comparisons test, where $* * * * \mathrm{p}<0.00001$ and $* \mathrm{p}<0.01$, when compared with the reference strain M2903 (F). AUC: area under the curve. L. braziliensis strains: M2903 (A); 346 (B); 426 (C); 330 (D); 340 (E). Data are shown as mean \pm SE (standard error of the mean) from three independent experiments performed in triplicates.

mediated agglutination favours $\mathrm{Sb}$ accumulation in isolate 346 (Fig. 3C), at least during early events of its exposure. To validate this observation, we performed similar experiments using a GPI-14 (manosyltransferase) Leishmania knockout strain (Ba262 $\Delta l p g$ ). GPI-14 is an essential enzyme in LPG assembling, and this knockout strain resulted in LPG absence in these mutants. As expected, increased $\mathrm{Sb}$ uptake was detected in LPG-deficient Leishmania Ba262 $\Delta l p g$ when compared with Ba262 wild-type after a $1 \mathrm{~h} \mathrm{Sb}$ incubation (Fig. 3D). A similar $\mathrm{Sb}$ accumulation pattern was observed for M2903 and 346 upon CA7AE Ab/Sb exposition (Fig. 3D).

\section{DISCUSSION}

Drug resistance is a multifactorial phenomenon, and efforts have been made to unravel common molecular markers of SbR strains. However, the nature of drug resistance together with biochemical heterogeneity among Leishmania species complicates this challenge. Because of that, the typing of $\mathrm{Sb}$ resistance based on multiple molecular markers identified in field isolates is relevant as a surveillance strategies to control leishmaniasis and to avoid $\mathrm{Sb}$ resistance spreading. As a wider study on the assessment of $\mathrm{Sb}$ resistance in Leishmania, this work focused on AT lesion-derived L. braziliensis, in which the $\mathrm{Sb}$ resistance index was at least 12 -fold when compared to the L. braziliensis reference strain. ${ }^{(3)}$
Because $\mathrm{Sb}$ resistance in Leishmania parasites usually involves drug transport modulation, in this study, we focused on the molecular and functional characterisation associated with $\mathrm{Sb}$ dynamics to mechanistically infer about this phenomenon in L. braziliensis clinical isolates. To achieve this goal, we started with the simplest and most cost effective functional way to indirectly evaluate AQP1 function; we added water to the Leishmania cell culture, which increased the cell volume, to monitor the osmoregulation-mediated recovery by absorbance. This technique has been used to infer about AQP1 function in trypanosomatids, ${ }^{(18)}$ and we found that cell recovery volume patterns upon hypo-osmotic shock were useful to distinguish between field and laboratory reference strain, M2903. This difference could be associated with adaptations under axenic culture conditions, because the L. braziliensis M2903 reference strain is very well adapted to in vitro maintenance compared to fresh (or fewer passages) clinical isolates. Once we determined that AQP1 was the main $\mathrm{Sb}$ entry route in Leishmania parasites ${ }^{(9)}$ and was also involved in osmoregulation, we decided to characterise the latter to determine AQP1 integrity and extrapolate to decreased uptake-related $\mathrm{Sb}$ resistance. However, despite the differences in recovery time, all parasites were able to re-establish cell volume - indirect evidence that AQP1 osmoregulation was not compromised. Nonetheless, the solute transport function can be impaired by 

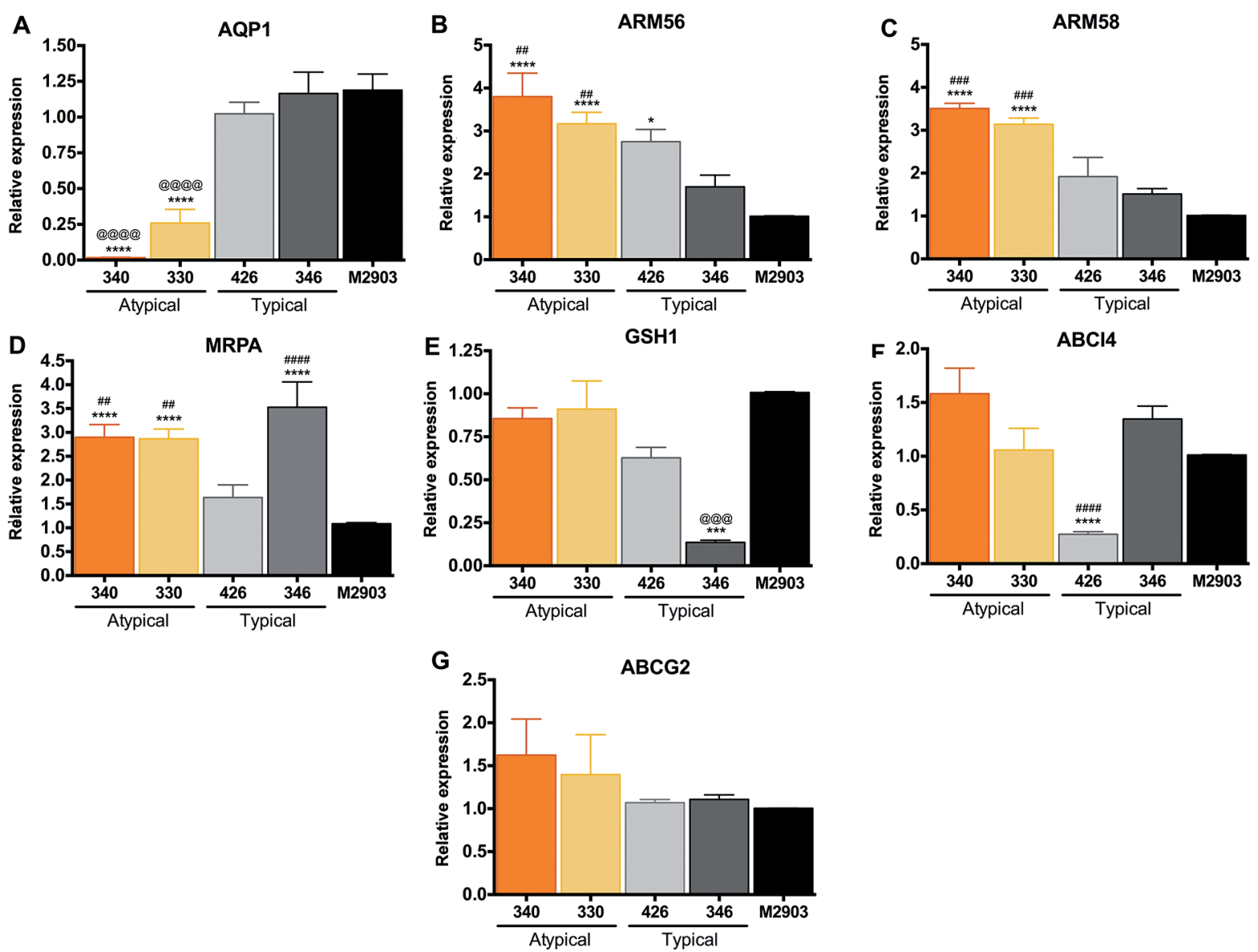

Fig. 2: transcript levels of antimony ( $\mathrm{Sb}$ ) resistance-associated genes in Leishmania braziliensis field isolates. (A) AQP1: aquaglyceroporin 1. (B) and (C) ARM56 and ARM58: antimony resistance marker of 56 and $58 \mathrm{kDa}$. (D) MRPA: multidrug resistant protein A (ABC transporter ABCC3, also known as PGPA). (E) GSH1: gamma-glutamylcysteine synthetase. (F) ABCI4: ATP-binding cassette protein subfamily I member 4. (G) ABCG2: ATP-binding cassette protein subfamily G, member 2. Constitutive GAPDH (LbrM.30.2950) was applied as housekeeping gene. Values represent the mean of at least two independent measurements performed with three biological replicates. Statistical analysis was performed using one-way analysis of variance (ANOVA) followed by Tukey's multicomparisons test. Asterisks represents the comparison with the M2903 reference strain, while \# or @ symbols indicates the differences between at least one of the SbR or Sb-sensitive strains. * $p<0.01$; $* * * \mathrm{p}<0.0002 ; * * * * \mathrm{p}<0.0001$.

specific point mutations and be associated with $\mathrm{Sb}$ resistance. ${ }^{(10)}$ Thus, we perform AQP1 gene sequencing, but we were unable to associate any SNPs to Sb resistance, because they were silent mutations, suggesting other mechanisms. However, exclusive SNPs on AQP1 from SbR or Sb-sensitive L. braziliensis (A516C and G447A) can be applied to the development of molecular diagnostic strategies that will be useful for drug resistance predictions.

Despite playing a role in osmoregulation, a reduction in AQP1 gene expression is associated with lower $\mathrm{Sb}^{\mathrm{III}} \mathrm{up}$ take, decreasing the drug concentration into Leishmania cells and resulting in a SbR phenotype. ${ }^{(10)}$ Because we were unable to associate $\mathrm{Sb}$ resistance with $\mathrm{AQP} 1$ mutations or compromised AQP1 osmoregulation-related function, we decided to verify its mRNA levels. Indeed, only SbR $L$. braziliensis field isolates 330 and 340 presented reduced AQP1 mRNA levels, which were functionally validated by reduced Sb uptake. AQP1 downregulation was also observed in other Leishmania species, such as L. tropica $^{(20)}$ and $L$. donovani, ${ }^{(21)}$ derived from patients unresponsive to
Sb-based treatment. Our data corroborate the reduced expression found not only in field isolates, but also in laboratory-SbR selected mutants. ${ }^{(10,19)}$ There are two studies on $L$. braziliensis isolated from Brazilian patients with different antimonial treatment outcomes showing either unchanged AQP1 levels ${ }^{(22)}$ or an increase in AQP1 expression. ${ }^{(23)}$ Eslami et al. also reported increased AQP1 levels in L. major isolated from patients refractory to glucantime in Iran. ${ }^{(24)}$ However, when citing previous work conducted by Dr. Rita Mukhopadhyay's team, ${ }^{(9)}$ Eslami et al. ${ }^{(24)}$ mistakenly affirmed that overexpression of $L m \mathrm{AQP} 1$ induces $\mathrm{Sb}$ resistance, which is not true and does not correspond to the findings by Gourbal et al., ${ }^{(9)}$ which observed a 10 -fold $\mathrm{Sb}^{\text {III }}$ resistance up to one allele $L m \mathrm{AQP} 1$ gene disruption. This raises doubts about how the authors in the work of Eslami et al. ${ }^{(24)}$ interpreted their data. Due to this mosaicism, it is important to establish species-specific markers once resistance is multifactorial. We clearly see an association between AQP1 downregulation in AT lesion-derived $L$. braziliensis isolates and reduced $\mathrm{Sb}$ uptake. 


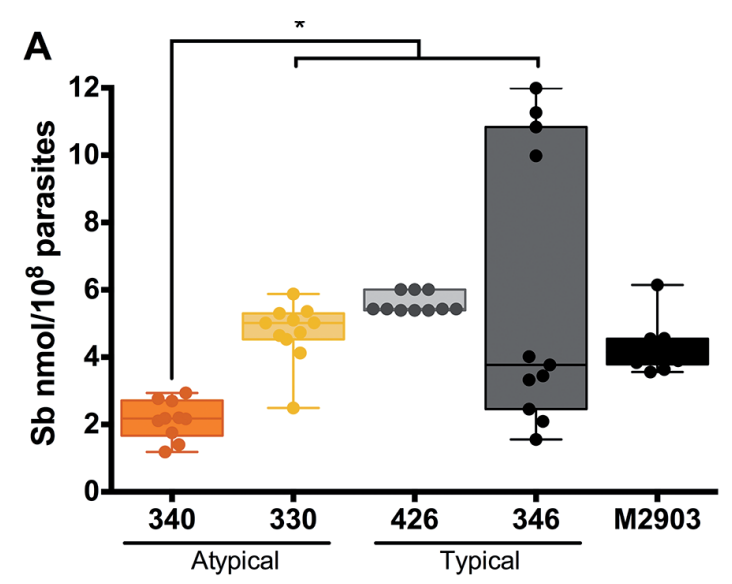

B
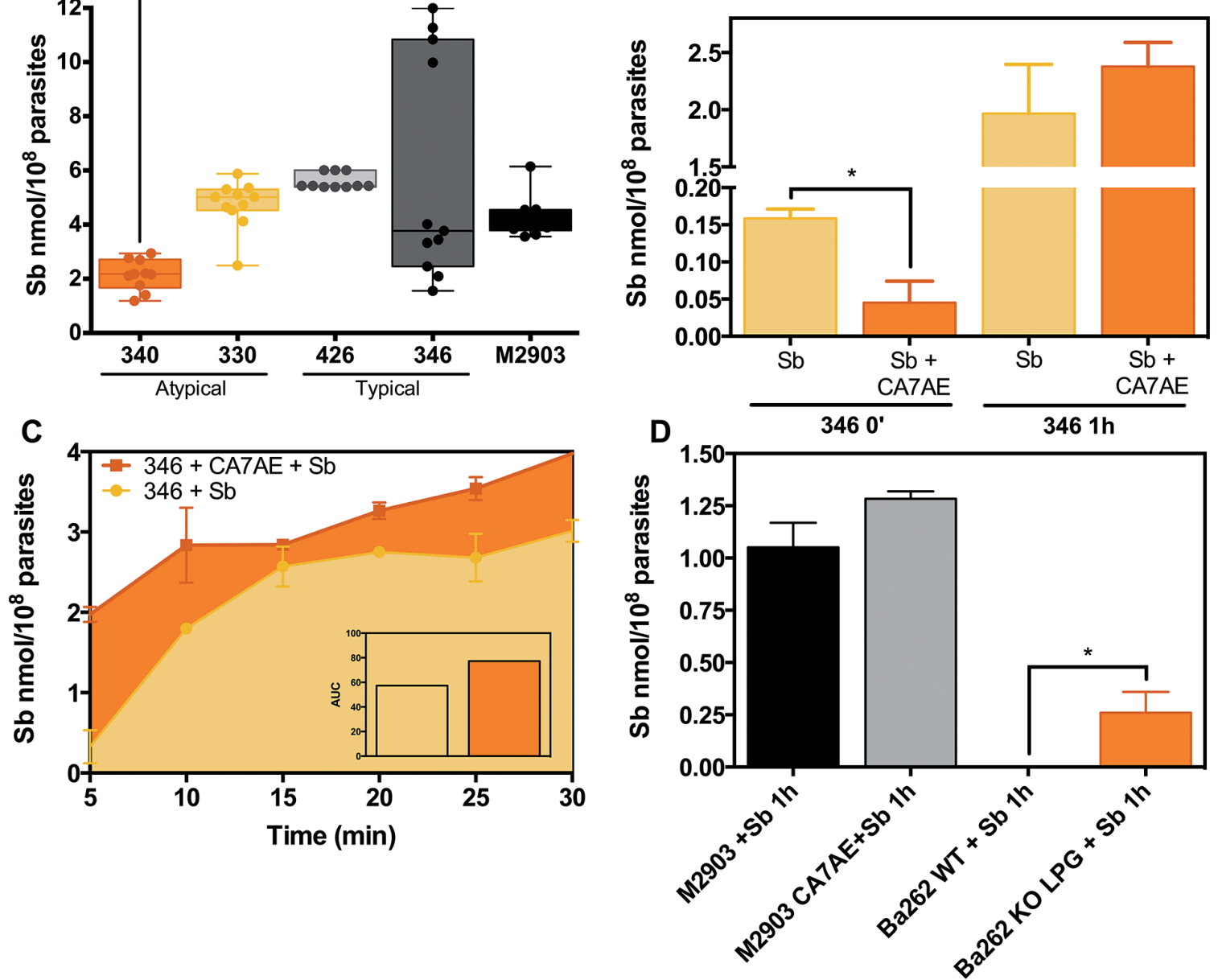

Fig. 3: antimony (Sb) uptake in Leishmania braziliensis field isolates. Mid-log phase promastigote forms were exposed to $500 \mathrm{mM}$ of $\mathrm{Sb} b^{\mathrm{III}}$ and incubated for $1 \mathrm{~h}$ at $26^{\circ} \mathrm{C}$ in HEPES/ NaCl/Glucose buffer. Cells were washed four times and digested in $65 \%$ nitric acid. Intracellular Sb traces were measured by graphite furnace atomic absorption spectrometry. Background matrix was established using only parasites without drug exposition. The average intracellular $\mathrm{Sb}$ value was subtracted from adsorption control - where $\mathrm{Sb}$ was added to the culture at $4^{\circ} \mathrm{C}$ and immediately washed with ice cold buffer - and normalised by $1 \times 10^{8}$ parasites. Sb uptake from both AT or typical lesion-derived L. braziliensis strains, $330,340,346$, and 426, was compared with reference strain M2903 (A). Sb uptake in L. braziliensis isolate 346 was assayed in the presence of CA7AE antibody, which recognises the Gal( $(\beta 1,4) \mathrm{Man}(\alpha 1)-\mathrm{PO}_{4}$ repeat units present in LPG, upon $1 \mathrm{~h}$ exposition $(\mathrm{B})$ or in a kinetic curve of early time points from 5 to $30 \mathrm{~min}(\mathrm{C})$. Ba262 KO LPG is a L. infantum strain where the mannosyltranferase encoding gene was disrupted, and they are not able to assemble LPG molecule (D). This strain was used as a control to infer about LPG interference in Sb uptake. *p $<0.01$. Data are shown as mean $\pm \mathrm{SE}$ (standard error of the mean) obtained from three independent experiments performed in quadruplicates.

Our next step was to compare mRNA levels of genes previously associated with $\mathrm{Sb}$ resistance. Because $\mathrm{Sb}$ resistance is a dynamic interplay between reduced drug uptake and increased efflux, we selected key transporters implicated in drug extrusion. The $\mathrm{ABC}$ transporter, MRPA (ABCC3), formerly known as P-glycoprotein A (PGPA), is responsible for the sequestration of metal-thiol conjugates to an intracellular organelle located near the flagellar pocket. ${ }^{(12)}$ MRPA is one of the most studied targets in SbR Leishmania and is found in both clinical isolates ${ }^{(12)}$ and laboratory selected mutants. ${ }^{(10)}$ In our study, MRPA mRNA levels were found to be upregulated in SbR and in typical lesion-derived Sb-sensitive L. braziliensis isolate 346 . This suggests that MRPA is not directly linked to $\mathrm{Sb}$ resistance in L. braziliensis obtained from Xakribá indigenous community in Minas
Gerais. ABC proteins, including ABCC3/MRPA, have thiols/Sb conjugates as substrates, promoting drug efflux. ${ }^{(8,12)}$ Intracellular $\mathrm{Sb}$ can also mediate the reduced thiol buffering capacity, inducing higher reduction environments by feedback towards drug resistance ${ }^{(8)}$ MRPA was increased in L. braziliensis isolate 346; however, $L$. braziliensis isolate 346 was the only isolate with downregulated GSH1 mRNA levels. This downregulation diminishes the functional relevance of MRPA upregulation. Reduced thiols can also support the Sb susceptibility phenotype observed in isolate 346 . Despite the previous link between transporters ABCI4/ABCG2 and $\mathrm{Sb}$ resistance, ${ }^{(14,15)}$ we were unable to confirm this phenotype in L. braziliensis clinical isolates. In fact, the $\mathrm{Sb}$-sensitive isolate 426 presented downregulation of ABCI4 mRNA levels. The link between ABCG2/ABCI4 
and $\mathrm{Sb}$ resistance was reported for L. major, ${ }^{(14,15)}$ which could be considered a species-specific feature. An observation that supports this hypothesis is the fact that AQP1 was upregulated in L. major clinical isolates. ${ }^{(24)}$

Recent studies headed by Dr Joachim Clos (BNITM, Germany) identified and functionally validated the overexpression of ARM56/ARM58 in SbR Leishmania spp. ${ }^{(16,25)}$ This overexpression was first identified by functional cloning using SbR libraries derived from L. braziliensis clinical isolates followed by heterologous expression and validation in L. infantum. ${ }^{(16,25)}$ ARM56 and ARM58 are part of a subtelomeric cluster on chromosome 34 and can be co-amplified in SbR parasites, favouring drug extrusion mediated by extracellular vesicles. ${ }^{(16)}$ Here, we identified increased ARM56/ARM58 transcripts in 'natural' SbR selected L. braziliensis, confirming their previous observation and reinforcing the usefulness of functional cloning based on libraries made from clinical isolates. The mechanistic way ARM56/ARM58 and AQP1 are regulated in L. braziliensis has yet to be described; however, because the gene regulation in trypanosomatids is post-transcriptional, the control relies on RNA processing, translation, and degradation. ${ }^{(26)}$ Drug pressure (or a given environmental selection) could trigger gene amplification or locus deletions that directly leads to increased or reduced/absent mRNA levels, respectively. ${ }^{(10)}$ These genomic rearrangements are a result of homology guided amplification of gene deletions. ${ }^{(27)}$ At the transcriptional level, RNA could be controlled by epigenetic markers, leading to increased mRNA steady state or promoting resection. ${ }^{(26)}$

In addition to molecular marker characterisation in $\mathrm{SbR}$ L. braziliensis, we also focused on $\mathrm{Sb}$ transport studies inspired by the higher intracellular $\mathrm{Sb}$ content in Sb-sensitive L. braziliensis isolate 346. We wonder if surface glycoconjugates would interfere with $\mathrm{Sb}$ uptake. Glycoconjugates play an important role in parasite virulence and infectiveness. The most abundant molecule in Leishmania glycocalyx is the LPG, abundant in promastigotes but downregulated in amastigote forms. ${ }^{(28,29)}$ LPG agglutination mediated by CA7AE seems to favour $\mathrm{Sb}$ uptake in parasites exposed up to $30 \mathrm{~min}$. After 1 $h$ exposure, the difference is no longer maintained and could be associated with LPG turnover time. The interference of LPG in Sb uptake was validated using a GPI14 knockout $L$. infantum Ba262 $\Delta l p g$ strain, which lacks LPG on its surface. ${ }^{(17)}$ Followed by $1 \mathrm{~h}$ treatment, Sb was detected only in the Ba262 mutant. The higher Sb uptake observed for L. braziliensis was in accordance with previous observations showing that LPG expression in this species is 10 - to 20 -fold lower than that in L. infantum. ${ }^{(30)}$ Thus, those preliminary observations provide evidence that surface glycoconjugates may interfere with $\mathrm{Sb}$ uptake, and further experiments are being performed.

In summary, simple molecular phenotyping, including drug transport studies and customised transcript level measurements, can be used to identify $\mathrm{Sb}$ resistance in Leishmania field isolates, helping determine treatment choice and outcome. Predicting Sb resistance in L. braziliensis field isolates could direct the chosen first-line treatment away from glucantime. Because drug resist- ance is a multifactorial phenomenon, different potential molecular markers must be combined and considered for the development of predictive assays applied to disease surveillance and to avoid resistance spreading. From all parameters analysed, we only observed a clear correlation in $\mathrm{Sb}$ resistance with higher expression of the ARM56/ ARM58 genes. However, other differences could also account for parasite variability. The limited sampling of only two isolates can be unrepresentative and not enough to stablish a pattern of markers for $\mathrm{Sb}$ resistance. It could also be species-specific or varies according to geographical regions, depending on where the isolates were obtained from (even classified as same species). Reduced AQP1 mRNA expression in combination with increased ARM58 mRNA and the A516C SNP in the AQP1 gene were sufficient to cluster the SbR clinical isolated L. braziliensis mutant. Altogether, these tools could be useful to predict treatment outcome and help control disease and resistance spreading. The next challenge is to apply these techniques to a greater number of isolates from different regions in order to validate these findings and to define the best markers for increasing therapeutic success.

\section{ACKNOWLEDGEMENTS}

To Dr Valéria Borges from Instituto Gonçalo Moniz, Fiocruz Bahia, Brazil, and Dr Albert Descouteaux from INRS - Institut Armand Frapier, Laval, Canada, for kindly providing the Leishmania infantum Ba262 $\Delta$ lpg strain. We thank the real-time PCR (RPT09D) and DNA sequencing (RPT01E) facilities at Instituto René Rachou/Fiocruz Minas.

\section{AUTHORS' CONTRIBUTION}

JNR, RPS and RLMN - Conceptualisation and experimental design; JNR and RLMN - investigated/performed experiments and analysed the data; CMFG and FF - contributed reagents/materials/analysis/tools; RLMN and RPS - supervision; RLMN - writing - original draft; JNR, CMFG, FF, RPS and RLMN - writing, review and editing.

\section{REFERENCES}

1. Guimarães LH, Machado PRL, Lago EL, Morgan DJ, Schriefer A, Bacellar O, et al. Atypical manifestations of tegumentary leishmaniasis in a transmission area of Leishmania braziliensis in the state of Bahia, Brazil. Trans R Soc Trop Med Hyg. 2009; 103(7): 712-5.

2. Guimarães LH, Queiroz A, Silva JA, Silva SC, Magalhães V, Lago EL, et al. Atypical manifestations of cutaneous leishmaniasis in a region endemic for Leishmania braziliensis: clinical, immunological and parasitological aspects. PLoS Negl Trop Dis. 2016; 10(12): e0005100.

3. Rugani JN, Quaresma PF, Gontijo CF, Soares RP, Monte-Neto RL. Intraspecies susceptibility of Leishmania (Viannia) braziliensis to antileishmanial drugs: antimony resistance in human isolates from atypical lesions. Biomed Pharmacother. 2018; 108: 1170-80.

4. Quaresma PF, de Brito CFA, Rugani JMN, Freire JM, Baptista RP, Moreno EC, et al. Distinct genetic profiles of Leishmania (Viannia) braziliensis associate with clinical variations in cutaneous-leishmaniasis patients from an endemic area in Brazil. Parasitology. 2018; 145(9): 1161-9.

5. Costa-Silva MF, Gomes LI, Martins-Filho OA, Rodrigues-Silva R, Freire JM, Quaresma PF, et al. Gene expression profile of cytokines and chemokines in skin lesions from Brazilian Indians with localized cutaneous leishmaniasis. Mol Immunol. 2014; 57(2): 74-85. 
6. Peters W. The treatment of kala-azar - new approaches to an old problem. Indian J Med Res. 1981; 73(Suppl.): 1-18.

7. Adaui V, Schnorbusch K, Zimic M, Gutiérrez A, Decuypere S, Vanaerschot $\mathrm{M}$, et al. Comparison of gene expression patterns among Leishmania braziliensis clinical isolates showing a different in vitro susceptibility to pentavalent antimony. Parasitology. 2011; 138(02): 183-93.

8. Wyllie S, Cunningham ML, Fairlamb AH. Dual action of antimonial drugs on thiol redox metabolism in the human pathogen Leishmania donovani. J Biol Chem. 2004; 279(38): 39925-32.

9. Gourbal B, Sonuc N, Bhattacharjee H, Legare D, Sundar S, Ouellette $\mathrm{M}$, et al. Drug uptake and modulation of drug resistance in Leishmania by an aquaglyceroporin. J Biol Chem. 2004; 279(30): 31010-7.

10. Monte-Neto R, Laffitte M-CN, Leprohon P, Reis P, Frézard F, Ouellette M. Intrachromosomal amplification, locus deletion and point mutation in the aquaglyceroporin AQP1 gene in antimony resistant Leishmania (Viannia) guyanensis. PLoS Negl Trop Dis. 2015; 9(2): e0003476.

11. Alijani Y, Hosseini SS, Ahmadian S, Boughattas S, Eslami G, Naderian S, et al. Molecular analysis of Aquaglyceroporin 1 Gene in non-healing clinical isolates obtained from patients with cutaneous leishmaniasis from Central of Iran. J Arthropod-Borne Dis. 2019; 13(2): 145-52.

12. Légaré D, Richard D, Mukhopadhyay R, Stierhof YD, Rosen BP, Haimeur A, et al. The Leishmania ATP-binding cassette protein PGPA is an intracellular metal-thiol transporter ATPase. J Biol Chem. 2001; 276(28): 26301-7.

13. Monte-Neto RL, Coelho AC, Raymond F, Légaré D, Corbeil J, Melo MN, et al. Gene expression profiling and molecular characterization of antimony resistance in Leishmania amazonensis. PLoS Negl Trop Dis. 2011; 5(5): e1167.

14. Perea A, Manzano JI, Castanys S, Gamarro F. The LABCG2 transporter from the protozoan parasite Leishmania is involved in antimony resistance. Antimicrob Agents Chemother. 2016; 60(6): 3489-96.

15. Manzano JI, Lecerf-Schmidt F, Lespinasse M-A, Di Pietro A, Castanys S, Boumendjel A, et al. Identification of specific reversal agents for Leishmania ABCI4-mediated antimony resistance by flavonoid and trolox derivative screening. J Antimicrob Chemother. $2014 ; 69(3)$ : 664-72.

16. Tejera Nevado P, Bifeld E, Höhn K, Clos J. A telomeric cluster of antimony resistance genes on chromosome 34 of Leishmania infantum. Antimicrob Agents Chemother. 2016; 60(9): 5262-75.

17. Lima JB, Araújo-Santos T, Lázaro-Souza M, Carneiro AB, Ibraim IC, Jesus-Santos FH, et al. Leishmania infantum lipophosphoglycan induced-Prostaglandin E2 production in association with PPAR- $\gamma$ expression via activation of Toll like receptors-1 and 2. Sci Rep. 2017; 7(1): 14321.
18. Rohloff P, Rodrigues CO, Docampo R. Regulatory volume decrease in Trypanosoma cruzi involves amino acid efflux and changes in intracellular calcium. Mol Biochem Parasitol. 2003; 126(2): 219-30.

19. dos Reis PG, do Monte-Neto RL, Melo MN, Frézard F. Biophysical and pharmacological characterization of energy-dependent efflux of Sb in laboratory-selected resistant strains of Leishmania (Viannia) subgenus. Front Cell Dev Biol. 2017; 5: 24.

20. Oliaee RT, Sharifi I, Afgar A, Kareshk AT, Asadi A, Heshmatkhah A, et al. Unresponsiveness to meglumine antimoniate in anthroponotic cutaneous leishmaniasis field isolates: analysis of resistance biomarkers by gene expression profiling. Trop Med Int Health. 2018; 23(6): 622-33.

21. Maharjan M, Singh S, Chatterjee M, Madhubala R. Role of aquaglyceroporin (AQP1) gene and drug uptake in antimony-resistant clinical isolates of Leishmania donovani. Am J Trop Med Hyg. 2008; 79(1): 69-75.

22. Torres DC, Adaui V, Ribeiro-Alves M, Romero GAS, Arévalo J, Cupolillo E, et al. Targeted gene expression profiling in Leishmania braziliensis and Leishmania guyanensis parasites isolated from Brazilian patients with different antimonial treatment outcomes. Infect Genet Evol. 2010; 10(6): 727-33.

23. Adaui V, Schnorbusch K, Zimic M, Gutiérrez A, Decuypere S, Vanaerschot $\mathrm{M}$, et al. Comparison of gene expression patterns among Leishmania braziliensis clinical isolates showing a different in vitro susceptibility to pentavalent antimony. Parasitology. 2011; 138(02): 183-93.

24. Eslami G, Zarchi MV, Moradi A, Hejazi SH, Sohrevardi SM, Vakili M, et al. Aquaglyceroporin1 gene expression in antimony resistance and susceptible Leishmania major isolates. J Vector Borne Dis. 2016; 53(4): 370-4.

25. Nühs A, Schäfer C, Zander D, Trübe L, Tejera Nevado P, Schmidt $\mathrm{S}$, et al. A novel marker, ARM58, confers antimony resistance to Leishmania spp. Int J Parasitol Drugs Drug Resist. 2014; 4(1): 37-47.

26. Clayton C. Regulation of gene expression in trypanosomatids: living with polycistronic transcription. Open Biol. 2019; 9(6): 190072.

27. Ubeda J-M, Raymond F, Mukherjee A, Plourde M, Gingras H, Roy G, et al. Genome-wide stochastic adaptive DNA amplification at direct and inverted DNA repeats in the parasite Leishmania. PLoS Biol. 2014; 12(5): e1001868.

28. Descoteaux A, Turco SJ. The lipophosphoglycan of Leishmania and macrophage protein kinase C. Parasitol Today (Regul Ed). 1993; 9(12): 468-71.

29. Assis RR, Ibraim IC, Noronha FS, Turco SJ, Soares RP. Glycoinositolphospholipids from Leishmania braziliensis and L. infantum: modulation of innate immune system and variations in carbohydrate structure. PLoS Neg1 Trop Dis. 2012; 6(2): e1543.

30. Soares RPP, Cardoso TL, Barron T, Araújo MSS, Pimenta PFP, Turco SJ. Leishmania braziliensis: a novel mechanism in the lipophosphoglycan regulation during metacyclogenesis. Int J Parasitol. 2005; 35(3): 245-53. 